\title{
Optimization of underground excavation in rock masses using ESO techniques
}

\author{
G. Ren \& Y. M. Xie \\ School of Civil, Environmental and Chemical Engineering, \\ RMIT University, Australia
}

\begin{abstract}
Excavation in rock masses induces complex stress redistribution around the opening. Inter alia, the stability of the opening is principally dependent on the stresses around the opening. The most influential factor attributing to the stress distribution is the geometrical shape of the opening. As such, finding the optimal shape for an underground excavation has practical significance in increasing stability and lowering support costs. This paper describes an approach to the shape optimization of underground excavation using Evolutionary Structural Optimization (ESO) techniques. Through illustrated examples, it is demonstrated that ESO method can be used to explore the excavation shapes that give optimized performance in terms of stress distribution and stability. The method is validated and compared with theoretical solutions. An example of a three dimensional case is presented in comparison with a well-known application case. Keywords: excavation, cavern, finite element, optimization, evolutionary structural optimization, numerical analysis.
\end{abstract}

\section{Introduction}

Stability is one of the primary considerations in an underground excavation design in rock masses. The stability of a rock structure is usually dependant on a number of factors, including the rock mass strength, supporting system and the shape of the excavation. In underground rock structure construction, the rock is used as a structural material which is subjected to massive stress regime change due to exaction. The shape of the excavation influences the stress distribution around the excavation, which in turn affects the stability of the excavation. In addition to satisfying the intended functional requirements of the excavation, it is important to select a propitiate excavation shape that gives the maximum 
stability. The shape optimization for an underground excavation in rock masses seeks to achieve the best performance based on stress regime change and the rock structural stiffness in a design domain.

The evolutionary structural optimization (ESO) techniques were originally developed by Xie and Steven in the 1990s [1], and recently were further developed into a Bi-directional evolutionary structural optimization (BESO) by Huang and Xie [2]. The original ESO method was based on the assumption that an optimal structure can be obtained by gradually removing all "inefficient materials" from the design domain. The "inefficient materials" are defined as those possess the least influence on either stress distribution or stiffness of the design domain. The design domain can be modelled by a finite element mesh with given boundary conditions and constraints. An example of such a finite element model is shown in Figure 1. The more recent bi-directional evolutionary structural optimization (BESO) method allows inefficient materials to be removed and at the same time to add new materials to the model. The BESO techniques significantly improve the efficiency of the optimization process. Both 2 or 3 dimensional models can be built for the optimization process using the ESO or BESO methods.

\section{Simulated verticalin-situ stress}

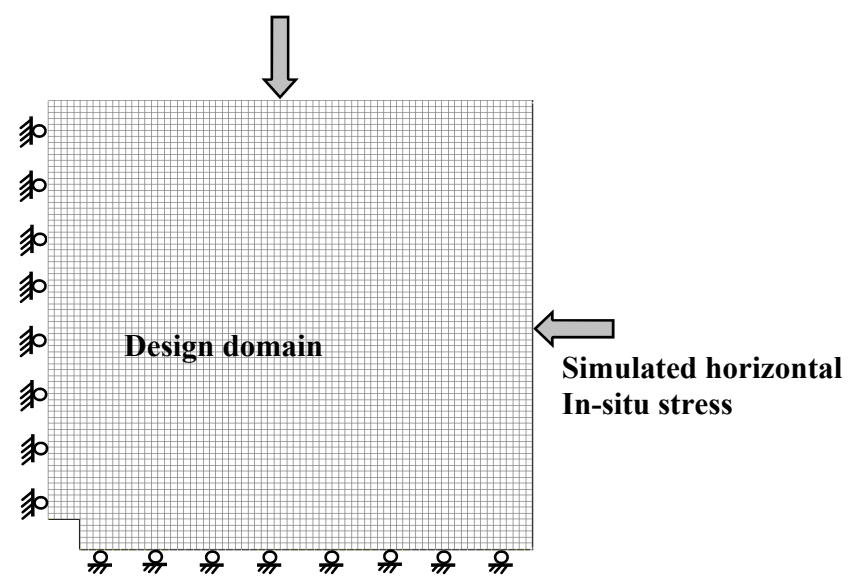

Figure 1: Two dimensional finite element mesh.

In the optimization process, an optimization criterion has to be used for determining the level of efficiency of each element. Both stress and stiffness can be used as criteria for the evolutionary optimization process. In the initial stage of the ESO/BESO procedure, a conventional finite element mesh is established to model the design domain. When stress is used as the optimization criterion, the ESO/BESO will evoke a routine to exam the stress levels of each element in the design domain. Elements with stresses below the predetermined stress level are recorded as inefficient elements and subsequently removed from the finite element mesh. As this evolutionary procedure is repeated for each updated mesh, 
more and more inefficient elements are removed from the mesh, so the remaining shape of the finite mesh evolves towards optimum. Full details of the ESO procedure using the stress criterion approach are described by Xie and Steven [1] and Ren et al. [3]. When stiffness is used as the optimization criterion, the ESO/BESO examines the effect of each element on the global stiffness. Elements in the design domain with low influence on the stiffness are deemed to be inefficient elements and are subsequently removed from the design domain (Huang and Xie [2]).

In 2004, Ren et al. [3] applied the ESO techniques to underground excavation and achieved interesting results. In an attempt to seek the optimal shape of an underground excavation, Ren et al. [3] adopted a full stress design strategy, i.e. the whole design domain is fully stressed. This is simulated in the finite element model by applying confined stresses equivalent to the vertical and horizontal insitu earth pressures (see Figure 1).

\section{Rock mass material model and optimization based on stresses around excavation}

Unlike manufactured materials, rocks are natural materials which can be highly variable. It is often practically difficult to adopt an appropriate constitutive material model for rock analysis, as the stress-train behaviour of rock is affected by its inherent geological discontinuities, such as beddings and faults. A number of material models for rock analysis have been developed over the years, the most commonly adopted material models include the conventional MohrCoulomb and Hoek-Brown models for the civil and mining industries. The earlier work on the shape optimization for underground excavation by Ren et al. [3] was limited to linear elastic model due to the limitation of computing power and ESO techniques available at the time. Recent advancement in PC computing power and the development in the BESO analytical efficiency have allowed for non-linear analysis to be carried out for better simulation of the rock mass behaviour.

In a traditional excavation design, a simple shape with an arched roof is usually adopted. It is always desirable to obtain a fully optimized shape within given constraints that would lead to stable excavation. Hoek and Brown [4] provided useful guidelines for underground excavation design based on stress distribution around certain excavation shapes.

Ren et al. [3] used the Von Mises stress as optimization criterion. At the initial stage of the optimization procedure, the finite element model was analyzed with an initiation void in the centre of the design mesh (Figure 1). The initiation void created initial stress change in the design domain so that the ESO routines could screen the stress levels in each element and apply an optimization criterion to eliminate those inefficient elements based on Von Mises stress:

$$
\sigma_{v m}=\sqrt{0.5 *\left[\left(\sigma_{1}-\sigma_{2}\right)^{2}+\left(\sigma_{2}-\sigma_{3}\right)^{2}+\left(\sigma_{3}-\sigma_{1}\right)^{2}\right]}
$$

where $\sigma_{1}, \sigma_{2}$ and $\sigma_{3}$ are principal stresses. 
The Von Mises stress $\left(\sigma_{v m}\right)$ was used in the optimization process as the optimization criterion. Elements which sustained a lower level of Von Mises stresses are regarded as inefficient in contributing to the global strength of the structure and hence were eliminated from the design domain. Some interesting shapes were produced using the Von Mises stress criterion approach. Figure 2 shows an example of optimized shape at an underground tunnel intersection. Figures 2(a), 2(b) and 2(c) illustrates the evolutionary process at iteration 15, 30 and 50 respectively.

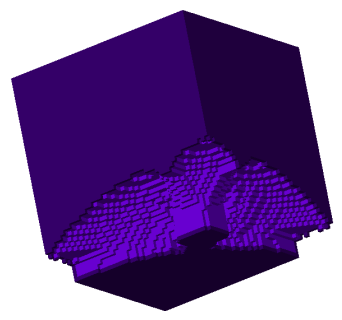

(a) Iteration 15

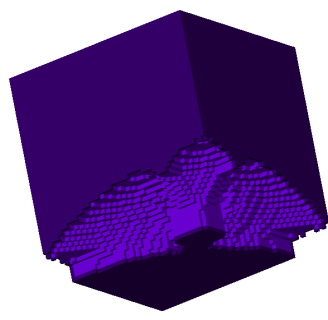

(b) Iteration 30

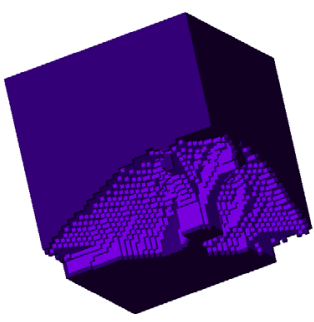

(c) Iteration 50

Figure 2: $\quad$ Example of evolution optimization process for a 3D finite element model.

\section{Optimization based on stiffness criterion}

When an excavation and its surrounding rock mass is considered as the design domain (Figure 3), the stiffness of the design domain is dependant of the shape of the excavation under the given constrains. The rationale behind the stiffness based optimization is that the higher global stiffness indicates a stable design domain, i.e. a stable rock structure. The optimization process aims to create a shape of excavation that would give the highest possible rock structure stiffness. Normally, the overall stiffness of a structure $K$ is inverse measure of the mean compliance $C$, which is defined by the total strain energy of the structure or the external work done by applied loads [2]:

$$
K u=f
$$

where $K$ is the global stiffness matrix; $f$ is the force vector and $u$ is the displacement vector.

If element $i$ is removed from the design domain, the stiffness matrix change is:

$$
\Delta K=K^{*}-K=K_{i}
$$


where $K^{*}$ is the stiffness matrix of the resulting structure after the element is removed and $K_{i}$ is the stiffness matrix of element $i$. The change in global stiffness as a result of the removal of element $i$ can be indexed by:

$$
\Delta C=\frac{1}{2} u_{i}^{T} K_{i} u_{i}
$$

where $u_{i}$ is the displacement vector of the element $i$.

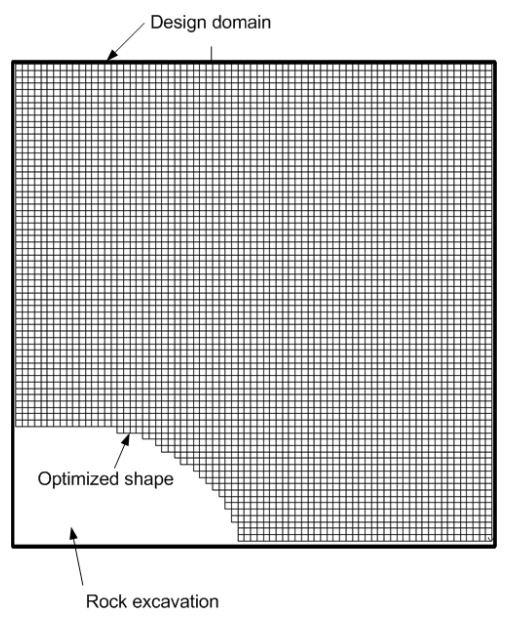

Figure 3: Stiffness optimization in rock mass excavation design domain (quarter model).

The equation (3) indicates the sensitivity of the mean compliance as a result of the removal of the element $i$, which equals to its elemental strain energy.

The evolutionary procedure for stiffness optimization is performed by checking the sensitivity of each element in the following way: elements in the close vicinity of the excavation with lower sensitivity as determined by Equation (3) would imply less efficiency in terms of contributing to the global stiffness; whereas, elements with higher sensitivity could be regarded as efficient elements for the rock structure. The optimization routines are designed to remove the lower efficiency elements in the finite element model in accordance with the predefined optimization criterion defined by Equation (3).

The process of removing elements in a finite element mesh is reminiscent of the underground excavation activity (see Figure 2), although the sequence of removal may be different. The optimized shape of the excavation is obtained when the pre-determined control parameter such as dimension of excavation or prescribed volume of removal is reached.

The full description and details of the ESO/BESO methodology are given in [1] and [5]. The following discussion will mainly focus on the application to underground excavation through illustrated examples. 


\section{Boundary conditions for underground excavation model}

For an underground excavation at depth of $z$, the vertical stress $\sigma_{\mathrm{v}}$ is given by:

$$
\sigma_{\mathrm{v}}=\gamma z
$$

where $\gamma$ is the unit weight of overlying stratum.

The simulated vertical stress as shown in Figure 1 can be defined using equation (4). The horizontal boundary stress $\sigma_{\mathrm{h}}$ can be estimated from:

$$
\sigma_{\mathrm{h}}=k \sigma_{\mathrm{v}}=k \gamma z
$$

where $k$ is the ratio of horizontal stress to the vertical stress.

The value of $k$ is affected by local geological conditions. Hoek et al. [6] suggested that the $k$ value for rock mass can be estimated by equation:

$$
k=0.25+7 E_{h}(0.001+1 / z)
$$

where $E_{h}$ in $\mathrm{GPa}$ is the average deformation modulus of the rock mass.

Equation (4) to (6) can be used to generate boundary stress conditions for the finite element models.

For a simple 2-D case, due to symmetry, only a quarter of the finite element is required to be analyzed as shown in Figure 1.

\section{Optimization model validation}

Under a conventional stress conditions such as the one shown in Figure 4, it is not hard to find a theoretical solution for the optimal shape of an excavation under vertical and horizontal stresses.

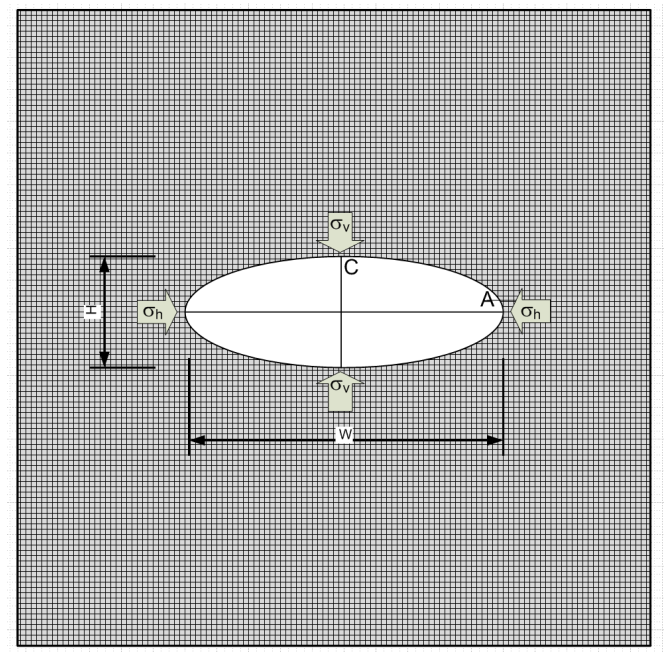

Figure 4: Optimal shape of an underground excavation is an elliptical opening with axial lengths matching the in situ stress ratio. 
In a biaxial stress field, the tangential boundary stresses $\left(\sigma_{A}\right.$ and $\left.\sigma_{C}\right)$ at the end of the axes of an elliptical opening (see Figure 4, where "W" denotes the length of the horizontal axis, " $\mathrm{H}$ " is the length of the vertical axis of the elliptical void) are given by equations:

$$
\begin{aligned}
& \sigma_{A}=\sigma_{y}+2 \sigma_{y}\left(\frac{W}{H}\right)-\sigma_{h} \\
& \sigma_{C}=\sigma_{h}+2 \sigma_{h}\left(\frac{H}{W}\right)-\sigma_{y}
\end{aligned}
$$

when $\sigma_{A}=\sigma_{C}$, we have:

$$
\frac{\sigma_{h}}{\sigma_{v}}=\frac{W}{H}
$$

It can be seen from equation (7) that the tangential boundary stresses are identical when the axis length ratio (aspect ratio) of an elliptical opening is matched to the in-situ stress ratio.

Figure 5 shows the optimized excavation shapes under various vertical and horizontal stress ratios, and Figure 6 illustrates the resulting optimized excavation for a 3 dimensional finite element model using the ESO procedure.

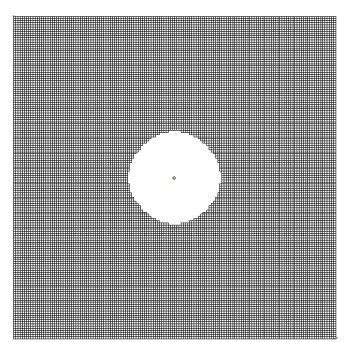

(a) Optimal shape for in-situ stress ratio 1:1

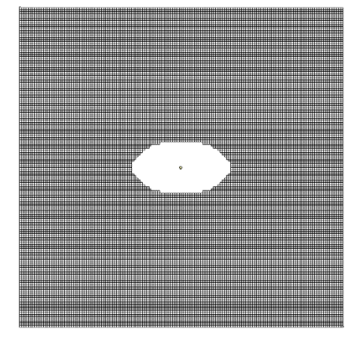

(b) Optimal shape for insitu stress ratio $\sigma_{y} / \sigma_{h}$ $=1 / 2$

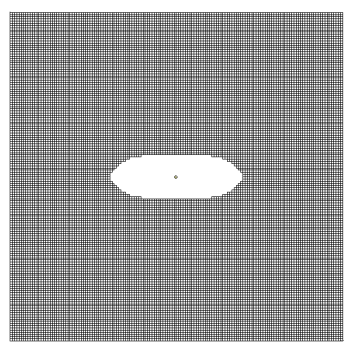

(c) Optimal shape for in-situ stress ratio $\sigma_{y} /$ $\sigma_{h}=1 / 3$

Figure 5: Optimal shapes obtained from ESO procedure under various stress ratios, indicating that the optimal shape for a given stress condition is elliptical opening with axial lengths matching the in-situ stress ratio.

From Figures 5 and 6, it is evident that the ESO derived shape gives identical answer to theoretical solutions, where the optimal shape of an excavation is in the shape of an oval with axis length ratio matching the in-situ stress ratio. 


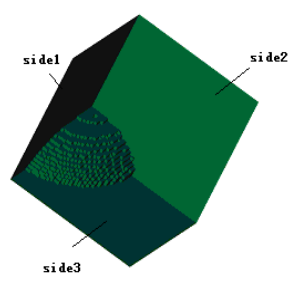

In situ stress ratio $3: 2: 1$ (Side1: 2: 3)

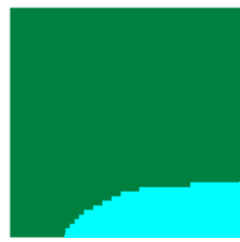

Side 1

Aspect ratio $=1: 3$

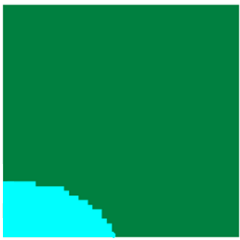

Side 2

Aspect ratio $=1: 2$

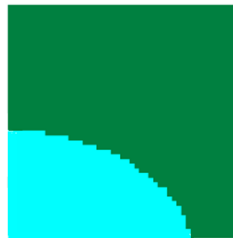

Side 3

Aspect ratio $=2: 3$

Figure 6: Optimized shape in 3 dimensional finite element models.

\section{Application examples and discussions}

To illustrate the applicability of the ESO/BESO techniques to underground excavation shape optimization, a model involving two excavations adjoining at a right angle are considered. This model can simulate tunnel intersection in rock masses. The 3-D model is subjected to vertical and horizontal confined stresses which can be represented by underground in-situ stresses. Figure 7 shows a processed image of the optimized shape of the excavation using Von Mises stress criterion. It is interesting to note that ESO/BESO method can generate rather practical configurations for an underground rock structure, which has close resemblance to one of the well-known Swedish Stockholm metro stations as shown in Figure 8 . The similarity between the model in Figure 7 and the Stockholm metro station as photographed in Figure 8 suggests that the rock structure design for the Stockholm metro station is not only aesthetically pleasing but also structurally optimal in terms of stress distribution and stability.

The ESO/BESO method is a useful tool in providing optimization solutions to real engineering problems with complex geometries and stress constrains. From the above discussion and demonstration, the following observations are made:

- The shape of excavation in rock mass can be optimized based on stress or stiffness of the rock structure.

- Optimized rock structures are theoretically more stable and require less support, and consequently lead to economical design.

- The ESO/BESO techniques are capable of handling complex rock structures and in-situ stress regimes.

- The ESO/BESO provides an alternative ways of forming an underground cavity based on prescribed constrains, such as vertical and horizontal stresses, function requirements, optimization criteria and material properties. 


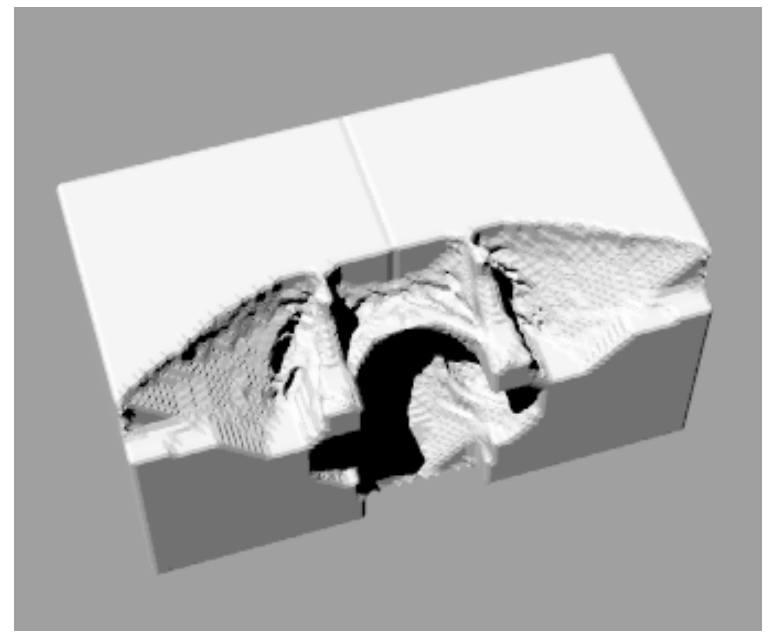

Figure 7: Optimized shape of an excavation at a tunnel intersection based on Von Mises stress criterion.

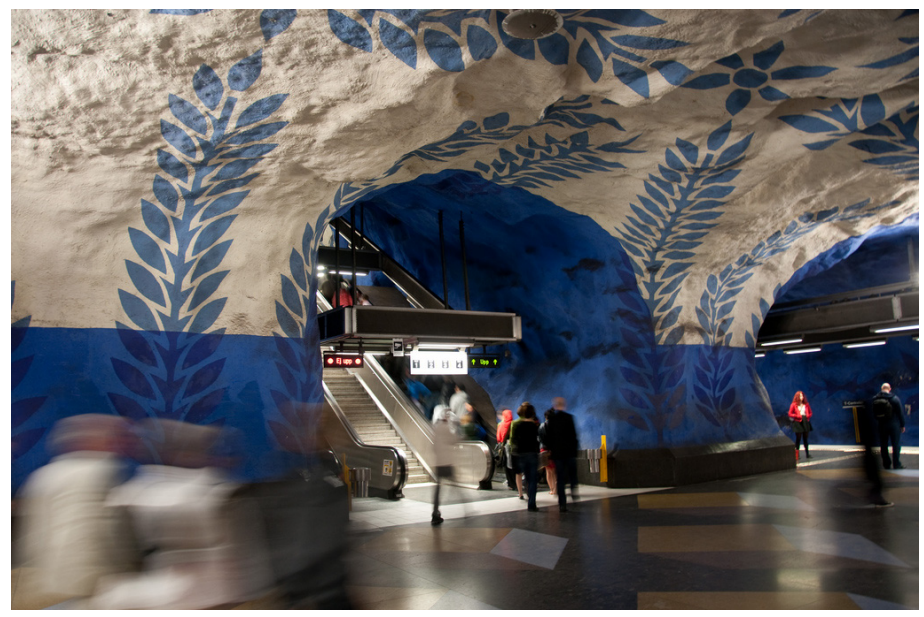

Figure 8: Stockholm metro system, Sweden is constructed in solid rock, a fine example of excavation in rock mass (photo courtesy of R. P. Young).

From a number of experiments, it is apparent that optimized shapes derived from the ESO/BESO method are optimization criterion dependent. The shape obtained using the Von Mises criterion is different from that using stiffness criterion. The meaning of "optimum shape" in the context of ESO/BESO may not be truly "the optimum" in strict mathematical sense; rather, it should be taken as the shape optimized to stress or stiffness according to the adopted 
criterion. More research is required as to which optimization criterion should be used for a specific design problem.

The significance of the ESO/BESO method applying to rock exaction lies in the fact that it is able to derive a geometrical shape that has been subjected to a filtering process eliminating inefficient elements in terms of stress or stiffness. The resulting shape of the excavation can be taken as the optimized one that gives better stability and consequently economical and safe underground rock structure.

\section{References}

[1] Xie, Y. M. and Steven, G. P., A simple evolutionary procedure for structural optimization, Springer, Berlin, 1997.

[2] Huang, X. and Xie, Y. M., Bi-directional evolutionary topology optimization of continuum structures with one or multiple materials, Computational Mechanics, Vol. 43, No. No. 3, 2008, pp.393-401.

[3] Ren, G., Smith, J. V., Tang, J. W., and Xie, Y. M., Underground excavation shape optimization using an evolutionary procedure, Computers and Geotechnics, Vol. 32, No. No. 2, 2005, pp.122-132.

[4] Hoek, E. and Brown, E. T., Underground excavations in rock, The Institute of Mining and Metallurgy London, 1980.

[5] Huang, X. and Xie, Y. M., Evolutionary Topology Optimization of Continuum Structures - Methods and Applications, John Wiley and Sons 2010.

[6] Hoek, E., Kaiser, P. K., and Bawden, W. F., Support of underground excavations in hard rock, Rotterdam: Balkema 1998. 